\section{Pocket guide to practical genetics}

Methodology in Medical Genetics: An Introduction to Statistical Methods. By Alan E. H. Emery. Pp. 157. (Churchill Livingstone: Edinburgh, London and New York, 1976.) £6.50.

THIS is a compact little book (I have reviewed it bit-by-bit, carrying it around in my pocket), clearly printed and well produced. The style is painstakingly explicit, missionary in tone, and unpretentious in its objectives and exposition.

The scope might fairly, and with no slight, be described as a compilation of those methods which Professor Emery, a practical human geneticist of extensive experience, has himself used. They comprise: standard segregation analysis (including some of the newer methods not covered in the classical texts); linkage analysis with and without the Bayesian modification; principles, but not details, of genetic counselling; twin studies; quantitative methods; and some of the elements of population genetics. There are many tables of wide utility, at present eclipsed by the computer, but (if our grants remain unfunded) likely to undergo a revival.

The author's unwavering practicality spares us the pages of pettifogging middle-grade mathematics which to the eyes of mathematicians and nonmathematicians alike, blight so many books on analytical genetics. His practicality is also a weakness. A really good mathematician with a true desire to communicate to the non-expert would have produced a somewhat more illuminating book. Here there are no underlying statistical principles stated: methods are produced with no visible means of support. Maximum likelihood estimation (MI.E) is used, but we are told nothing of its properties, a neglect which has its counterpart in some of the methods chosen. For instance, there seems scarcely any point now in the Lenz-Hogben method which has never been well defended (for example, as to the asymptotic normality of the test statistic), is not based on a sufficient statistic, and falls far short of maximal power. On the other hand the MLE method recommended for the frequency of a rare recessive gene is so badly biased as to merit a jack-knife correction: in small samples it far from follows a normal distribution, a property implicitly invoked on page 5. (A Poisson approximation with confidence limits based on the $\chi^{2}$ distribution would have been preferable.) Again MLE of $\theta$, the recombinant coefficient, is so biased for large $\theta$ as to make estimation of loose linkage hazardous in anything but immense samples: thus it scarcely matters what, if any, mapping function is used.

These defects are likcly to leave the student, nurtured on this hook alone. with little statistical sense and without either inventiveness or resourcefulness. This trend shows up in the method recommended for estimating the frequency of an X-linked recessive gene, which discards all information on female subjects. (Numerical MLE is straightforward, if a trifle inelegant.) The $\triangle B O$ problem is dealt with by Bernstein's method, which is absolutely. efficient asymptotically but which would not be so for four alleles or more.

\section{Integrated text on population biology}

Population Biology. By Thomas C.. Emmel. Pp.xii + 371. (Harper and Row: New York and London, 1976.) £11.95: $\$ 15.95$.

As the author remarks in his preface, "part of the problem of writing a book on population biology involves the host of inter-relationships among the basic contributing ficlds of ecology, genctics, evolution and bchaviour". Dr I:mmel copes well with this problem, providing a clearly arranged up-to-date account for the "well-prepared, advanced undergraduate". The book would also serve the professional biologist needing to catch up with some aspects of this recently integrated subject.

The chapters deal with cycles of matter and energy, population genetics and evolution, population dynamics. dispersion, dispersal. age and sex structure of populations, life-history patterns, mating systems, behaviour. climatic effects, and interactions of populations in communities. The reference list of 1.400 or so items is strong on recent contributions, and the index is reasonably comprehensive. The illustrations are on the whole excellent, although a few of the line drawings are disproportionately tall, and several of the half-tone pictures fall short of their purpose the zebras in 11-14 do not appear cryptic, whereas the cicadas in $8-2$ b do (but should not).

In a fairly advanced book presenting many modern idcas and examples. workers in this ficld will inevitably find things to disagrec with, and also errors. We are told on p222 that populations emphasising $r$ selection typically inhabit regions with variable climates. such as the temperate zones, and on
I am afraid, however, that few biologists are mathematically adventurous. No doubt Professor Enery knows his audience at least as well as I do and caters for them appropriately. With a good, well-informed, teacher as a safeguard, this book might be successfully used as an elementary text. and many more sophisticated geneticists would be happy to have it in their pockets for ready reference.

Edmond A. Murphy

Edmond A. Murphy is Professor of Medicine at Johns Hopkins University, and Director of the Division of Medical (ienetics at the Johns Hopkins University school of Medicine, Baltimore, Maryland.

p224 that most terrestrial vertebrates and perennial plants seem to be $K$ selected. whereas most annual plants and insects are apparently $r$ selected. The joint consequences of these two generalisations might well startle a biogeographer. Only later are we warned of the uncertainties of $r$ and $K$ selection theory.

Most of the errors I noticed were minor misprints, an omission of a text citation from the reference list, and so on. I regard the original meaning of "decimate" and the proper spelling of "rcpellants" (noun) as lost causes, but hope we may save Rhagoletis from being confused $(p 60)$ with the "frit" flies. More serious is the confusing blunder in Fig. 5-16. in which the prey curve is labelled "predator". and vice" versa. On 2229 , the net reproduction rate $R_{n}$ is said to be the number of surviving offspring per female: it should be female offspring. On p.53. one of the equations for intensity of natural selection is badly misprinted.

Although the material. like the subject itself. is predominantly American, examples are taken from many parts of the World, including Britain. Some of the examples cited are difficult to present briefly, and in places (for example. ppl37-8) the exposition is not clear enough.

To a British reader it seems odd to find no mention of life-table analysis and $k$ factor analysis for diagnosing 'key factors' and density relationships among the successive mortalitics in the life-cycle. If this (as it seems to $m e)$ is a serious omission. it is an exceptional feature in this generally comprehensive and well-balanced account. With its stimulatingly up-to-date treatment. I should think it worthy of a place in the short-list of hooks for students of population biology.

M. E. Solomon

M. E. Solomon is Head of the Zoology Se'ction at Long Ashton Research Station, University of Bristol UK 